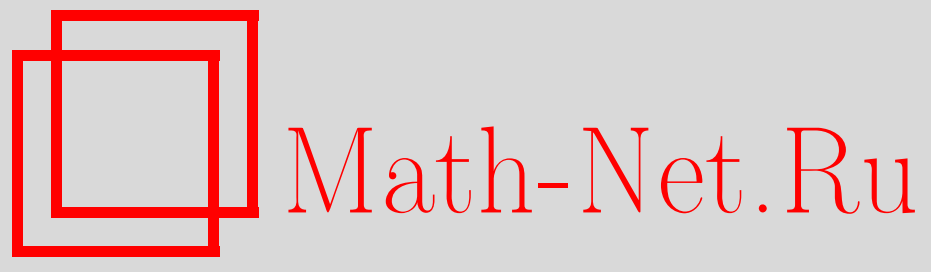

В. С. Рябенький, Разностные потенциалы, аналогичные интегралам Коши, УМH, 2012, том 67, выпуск 3, 147-172 DOI: https://doi.org/10.4213/rm9483

Использование Общероссийского математического портала Math-Net.Ru подразумевает, что вы прочитали и согласны с пользовательским соглашением http://www . mathnet.ru/rus/agreement

Параметры загрузки:

IP : 35.174 .16 .151

26 апреля 2023 г., 17:02:29

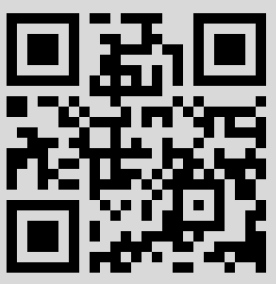




\section{Разностные потенциалы, аналогичные интегралам Коши}

\section{В. С. Рябенький}

Излагается современное состояние теории потенциалов для решений систем линейных разностных уравнений, предложенной автором в 1969 г. Впервые подробно прослеживается аналогия между той ролью, которую разностные потенциалы играют для решений линейных разностных схем общего вида, и той ролью, которую интегралы типа Коши играют для аналитических функций. Излагается существо новых возможностей, доставляемых теорией разностных потенциалов и возникающих благодаря объединению универсальности и алгоритмичности разностных схем с некоторыми свойствами интегралов типа Коши. Дается краткий библиографический обзор некоторых основных из уже реализованных приложений теории.

Библиография: 61 название.

Ключевые слова: разностные потенциалы, интегралы Коши, численное решение краевых задач, искусственные граничные условия, математическая теория управления звуком.

\section{СОДЕРЖАНИЕ}

1. Определение абстрактных линейных разностных схем............. 147

2. Абстрактные разностные схемы в составных сеточных областях ...... 151

3. Определение и свойства разностных потенциалов ............... 153

4. Аналогия между разностными потенциалами и интегралами Коши ... 163

5. Теория разностных потенциалов как база для некоторых приложений . 166

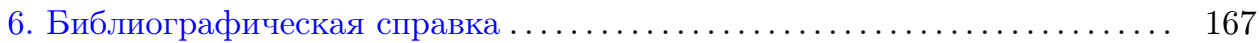

Список литературы . . . . . . . . . . . . . . . . . . . . . . . . 168

\section{1. Определение абстрактных линейных разностных схем}

Абстрактной разностной схемой будем называть произвольную систему линейных разностных уравнений, записанную в виде

$$
\sum_{n \in N_{m}} a_{m n} u_{n}=f_{m}, \quad m \in M,
$$

где $M$ - произвольное конечное множество абстрактных индексов $m \in M ; f_{m}-$ вектор с числовыми компонентами, количество которых может зависеть от $m$;

Работа выполнена при поддержке РФФИ (грант № 11-01-00114).

(C) В. С. Рявенький, 2012 
$N_{m}$ - конечное множество абстрактных индексов $n \in N_{m} ; u_{n}$ - вектор с числовыми компонентами, количество которых может зависеть от $n ; a_{m n}, m \in M$, $n \in N_{m},-$ прямоугольные матрицы, размерности которых согласованы с размерностью вектора $f_{m}$ и векторов $u_{n}, n \in N_{m}$, так, чтобы равенство (1.1) имело смысл. Область определения решения системы (1.1) есть, очевидно, конечное множество $N=\bigcup_{m \in M} N_{m}$ абстрактных индексов $n \in N$. Обозначим через $F_{M}$ и $V_{N}$ линейные пространства всех вектор-функций $f_{M}=\left\{f_{m}, m \in M\right\}$ и всех вектор-функций $u_{N}=\left\{u_{n}, n \in N\right\}$ соответственно. Запись системы линейных разностных уравнений в форме (1.1) будем называть абстрактной разностной схемой. Множества $M$ и $N$ абстрактных индексов будем называть сетками, на которых задана разностная схема, а множество $N_{m}$ - шаблоном разностной схемы в точке $m \in M$. K рассматриваемым разностным схемам (1.1) будем предъявлять только следующее требование: разностная схема (1.1) имеет одно и только одно решение $u_{N} \in V_{N}$ при любой правой части $f_{M} \in F_{M}$.

Система линейных алгебраических уравнений, записанная в канонической форме

$$
\sum_{j=1}^{s} b_{i j} x_{j}=\varphi_{i}, \quad i=1,2, \ldots, s,
$$

с матрицей $B=\left\{b_{i j}\right\}$, $\operatorname{det} B \neq 0$, может быть записана в форме разностной схемы (1.1). Достаточно принять за $M$ множество целых чисел $1,2, \ldots, s$. За $N_{m}$ при каждом $m=1,2, \ldots, s$ принять то же самое множество $M$, одно и то же при каждом $m \in M$. Множество $N=\bigcup N_{m}, m \in M$, в таком случае тоже совпадает с множеством $M$. Функциями $f_{M}$ и $u_{N}$ в схеме (1.1) в случае (1.2) будут соответственно числовые функции $\varphi_{m}, m=1,2, \ldots, s$, и $u_{n}, n=1,2, \ldots, s$. Коэффициентами $a_{m n}$ при записи системы (1.2) в форме разностной схемы $(1.1)$ будут числа $a_{m n}=b_{m n}, m, n=1,2, \ldots, s$.

Излагаемая ниже формальная теория разностных потенциалов, аналогичных интегралам Коши, ничего не добавляет к теории систем линейных разностных уравнений общего вида (1.2). Однако формальная теория разностных потенциалов, подобных интегралу типа Коши, становится содержательной в случаях, если матрица системы (1.2) сильно разрежена и эта конкретная специфика разреженности соответствующим (должным) образом учтена в записи системы (1.2) в форме абстрактной разностной схемы (1.1). В частности, использование записи системы линейных уравнений в форме абстрактной разностной схемы (1.1) естественно, а формальная теория разностных потенциалов дает новые возможности в случае классических разностных схем, аппроксимирующих линейные стационарные краевые задачи или нестационарные начально-краевые задачи для уравнений с частными производными. Приведем примеры записи классических разностных схем в канонической форме (1.1).

ПримеР 1. Построим разностную аппроксимацию задачи Дирихле для уравнения Пуассона в квадратной области $D:\left\{\left|x_{1}\right|<1,\left|x_{2}\right|<1\right\}$ с границей $\partial D$ :

$$
\begin{aligned}
\frac{\partial^{2} u}{\partial x_{1}^{2}}+\frac{\partial^{2} u}{\partial x_{2}^{2}} & =\varphi\left(x_{1}, x_{2}\right), & & \left(x_{1}, x_{2}\right) \in D \\
\left.u\right|_{\partial D} & =\psi\left(x_{1}, x_{2}\right), & \left(x_{1}, x_{2}\right) & \in \partial D .
\end{aligned}
$$


Зададим шаг $h>0, h^{-1}$ - целое число, и построим сетку $M$ точек $m=$ $\left(m_{1} h, m_{2} h\right),\left|m_{1}\right| \leqslant h^{-1},\left|m_{2}\right| \leqslant h^{-1}$, исключая четыре угловые точки квадрата $D$. Каждой точке сетки $m \in M$, лежащей внутри квадрата $D$, поставим в соответствие шаблон, состоящий из пяти точек $n$ :

$$
N_{m}=\left\{\left(m_{1}, m_{2}\right),\left(m_{1} \pm 1, m_{2}\right),\left(m_{1}, m_{2} \pm 1\right)\right\}, \quad m \in D .
$$

Мы пишем $m=\left(m_{1}, m_{2}\right)$ вместо $m=\left(m_{1} h, m_{2} h\right)$ для краткости. Зададим коэффициенты $a_{m n}, m \in M, n \in N_{m}$, формулами

$$
a_{m n}= \begin{cases}-4 h^{-2}, & \text { если } n=\left(m_{1}, m_{2}\right), \\ h^{-2}, & \text { если } n=\left(m_{1} \pm 1, m_{2}\right) \text { или } n=\left(m_{1}, m_{2} \pm 1\right) .\end{cases}
$$

Каждой точке $m \in M$, лежащей на границе $\partial D$ квадрата, кроме четырех угловых, поставим в соответствие шаблон $N_{m}$, состоящий из одной точки, совпадающей с точкой $m$ :

$$
N_{m}=n=m, \quad m \in \partial D
$$

Зададим коэффициент $a_{m n}$ в этом случае, т. е. в случае $m \in \partial D, n \in N_{m}$, положив

$$
a_{m n}=a_{m m}=1
$$

Далее, положим

$$
f_{m}= \begin{cases}\varphi_{m}, & \text { если } m \in D, \\ \psi_{m}, & \text { если } m \in \partial D,\end{cases}
$$

где $\varphi_{m}=\varphi\left(m_{1} h, m_{2} h\right), \psi_{m}=\psi\left(m_{1} h, m_{2} h\right)$.

Множество $N=\bigcup_{m \in M} N_{m}$ совпадает в этом примере с множеством $M$. Простейшая пятиточечная разностная аппроксимация задачи (1.3), (1.4), построенная выше, запишется при выбранных обозначениях в виде (1.1).

Пример 2. Рассмотрим вместо задачи (1.3), (1.4) для уравнения Пуассона задачу с другим краевым условием на стороне $x_{1}=-1$ квадрата $D$ и с теми же условиями Дирихле на остальных трех сторонах $\partial D$ квадрата $D$ :

$$
\begin{gathered}
\left.\frac{\partial u}{\partial x_{1}}\right|_{x_{1}=-1}=\left.\psi\left(x_{1}, x_{2}\right)\right|_{x_{1}=-1}, \quad \text { если } x_{1}=-1, \\
\left.u\right|_{x=\left(1, x_{2}\right)}=\psi\left(1, x_{2}\right),\left.\quad u\right|_{x=\left(x_{1}, \pm 1\right)}=\psi\left(x_{1}, \pm 1\right) .
\end{gathered}
$$

При построении аппроксимирующей задачу (1.3), (1.5) разностной схемы множество $M$ возьмем то же, что и в примере 1 . Каждой точке $m \in M$ кроме точек, лежащих на стороне $x_{1}=-1$ квадрата $D$, поставим в соответствие тот же шаблон $N_{m}$ и те же коэффициенты $a_{m n}$, что и в примере 1 . Точкам $m \in M$, лежащим на стороне $x_{1}=-1$ квадрата $D$, поставим в соответствие двухточечный шаблон $N_{m}=\left\{\left(-1, m_{2} h\right),\left(-1+h, m_{2} h\right)\right\}$, и коэффициенты

$$
\begin{array}{ll}
a_{m n}=h^{-1}, & \text { если } n=\left(-1+h, m_{2} h\right), \\
a_{m n}=-h^{-1}, & \text { если } n=\left(-1, m_{2} h\right) .
\end{array}
$$


Выбор $N_{m}$ и коэффициентов $a_{m n}$ по формуле (1.6) означает, что мы аппроксимировали первое из условий (1.5), заменив производную $\frac{\partial u}{\partial x_{1}}$ простейшим разностным отношением первого порядка. Сетка $N=\bigcup_{m \in M} N_{m}$, как и в примере 1 , совпадает с сеткой $M$. Значения $f_{m}, m \in M$, определим той же формулой, что и в примере 1 .

При сделанных обозначениях построенная простейшая пятиточечная разностная схема, аппроксимирующая задачу (1.3), (1.5), запишется в виде (1.1).

В примерах 1 и 2 сетки $M$ и $N$ совпадают: $M=N$. Приведем пример записи традиционной разностной схемы на разнесенных сетках в виде (1.1), в котором $M \neq N$.

ПримеР 3. Выберем $h>0, h^{-1}$ - целое число, и рассмотрим разностный аналог задачи

$$
\begin{gathered}
\frac{d u}{d x}+a u=\varphi(x), \quad 0<x<1, \\
\left.u\right|_{x=0}=\psi,
\end{gathered}
$$

а именно разностную задачу

$$
\begin{gathered}
\frac{u_{m+1}-u_{m}}{h}+a \frac{u_{m+1}+u_{m}}{2}=\varphi_{m+1 / 2}, \quad 0<\left(m+\frac{1}{2}\right) h<1, \\
u_{0}=\psi .
\end{gathered}
$$

Определим сетку точек $M$, отнеся к этой сетке все "полуцелые" точки $m$ с координатами $x_{m+1 / 2}=(m+1 / 2) h, 0<x_{m+1 / 2}<1$, а также точку $m=0$ с координатой $x_{0}=0 h=0$.

Поставим в соответствие каждой полуцелой точке $m \in M$, с координатой $x_{m}=(m+1 / 2) h$ шаблон $N_{m}$, состоящий из двух точек $n$ с координатами $x_{n}=m h$ и $x_{n}=(m+1) h$, положив $N_{m}=\{n=m h, n=(m+1) h\}$. Точке $m=0$ с координатой $x_{0}=0 h=0$ поставим в соответствие шаблон $N_{0}$, состоящий из одной точки $N_{0}=0 h$.

Тогда множество $N=\bigcup_{m \in M} N_{m}$ состоит из всех "целых" точек $n=0$, $h, \ldots, 1-h, 1$, лежащих на отрезке $0 \leqslant x \leqslant 1$. Коэффициенты $a_{m n}$ определим равенствами

$$
\begin{array}{llrl}
a_{m n}=\frac{1}{h}+\frac{a}{2}, & \text { если } x_{m}=\left(m+\frac{1}{2}\right) h, & x_{n}=(m+1) h \in N_{m}, \\
a_{m n}=-\frac{1}{h}+\frac{a}{2}, & \text { если } x_{m}=\left(m+\frac{1}{2}\right) h, & x_{n}=(m-1) h \in N_{m}, \\
a_{m n}=1, & \text { если } x_{0}=0 h, & x_{n}=x_{0}=0 \in N_{m} .
\end{array}
$$

Положим $f_{m}=\varphi_{m}$, если $m$ - полуцелая точка, и $f_{0}=\psi$.

При сделанных обозначениях разностная задача (1.7) запишется в каноническом виде (1.1). 
Можно привести к каноническому виду (1.1) разностные краевые задачи, аппроксимирующие линейные краевые задачи для уравнений с частными производными на нерегулярных сетках в многомерном пространстве, в областях с разрезами, для уравнений на сфере или другом многообразии, для краевых задач в случае систем разностных линейных разностных уравнений.

\section{2. Абстрактные разностные схемы в составных сеточных областях}

Зададим произвольно $M^{+} \subset M$. Введем следующие обозначения: $M^{-}=$ $M \backslash M^{+}, N^{+}=\bigcup_{m \in M^{+}} N_{m}, N^{-}=\bigcup_{m \in M^{-}} N_{m}, \gamma=N^{+} \cap N^{-}$. Множество $\gamma$ будем называть сеточной границей между сеточными подобластями $N^{+}$и $N^{-}$ составной области $N=N^{+} \cup N^{-}$.

Введем линейное пространство $V_{\gamma}$ всех тех функций $u_{\gamma}$, которые являются сужениями $\left.u_{N}\right|_{\gamma}$ функций $u_{N} \in V_{N}$ на подмножество $\gamma=N^{+} \cap N^{-}$множества $N$.

Наряду с абстрактной схемой (1.1) рассмотрим следующие две абстрактные разностные схемы:

$$
\begin{aligned}
& \sum_{n \in N_{m}} a_{m n} u_{n}^{-}=\theta_{M}\left(M^{+}\right) f_{m}, \quad m \in M, \\
& \sum_{n \in N_{m}} a_{m n} u_{n}^{+}=\theta_{M}\left(M^{-}\right) f_{m}, \quad m \in M .
\end{aligned}
$$

Здесь $\theta_{Y}(X), X \subset Y,-$ характеристическая функция подобласти $X$ области $Y$. Очевидно, что решение $u_{N}=\left\{u_{n}\right\}$ задачи (1.1) связано с решениями $u_{N}^{-}$ и $u_{N}^{+}$задач $(2.1)$ и $(2.2)$ равенством

$$
u_{N}=u_{N}^{-}+u_{N}^{+}
$$

или, подробнее,

$$
u_{n}=u_{n}^{-}+u_{n}^{+}, \quad n \in N .
$$

Решение $u_{N}^{+}$задачи (2.2) будем называть вкладом влияния источников $\theta_{M}\left(M^{-}\right) f_{m}$, локализованных на $M^{-}$, в решение $u_{N}$ задачи (1.1), а $u_{N}^{-}$вкладом влияния источников $\theta_{M}\left(M^{+}\right) f_{m}$ в решение $u_{N}$, локализованных на $M^{+}$, той же задачи (1.1).

Примеры задач в составных областях. Воспользуемся разностной краевой задачей из примера 1 в разделе 1 и рассмотрим три примера составных областей. В первом примере отнесем к подмножеству $M^{+} \subset M$, все те точки $m \in M$, которые попали внутрь или на границу Г подобласти $D^{+}$квадрата $D$, изображенной на рис. 1. Во втором примере отнесем к $M^{+}$те точки $m \in M$, которые попали внутрь или на границу Г несвязной области $D^{+}=D_{1}^{+} \cup D_{2}^{+}$, изображенной на рис. 3. На рис. 5 граница раздела Г выходит на границу $\partial D$ области $D$. В этом случае к $M^{+}$отнесем точки $m \in M$, попавшие в область $D^{+}$ или на ее границу $\Gamma$.

После того как $M^{+}$выбрано, автоматически определятся подмножества $M^{-}$; $N^{+}=\bigcup_{m \in M^{+}} N_{m}, N^{-}=\bigcup_{m \in M^{-}} N_{m}$ и граница $\gamma=N^{+} \cap N^{-}$между подобластями $N^{+}$и $N^{-}$. 


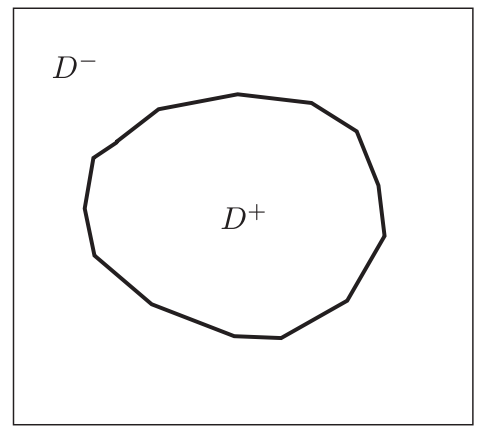

Рис. 1

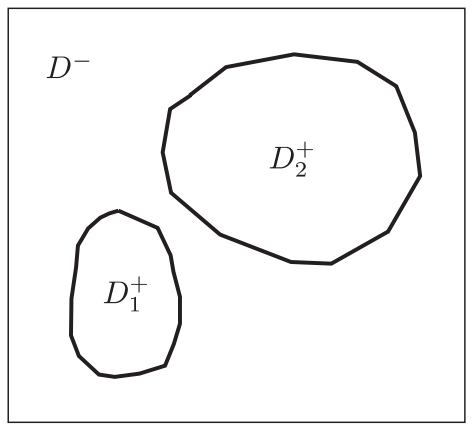

Рис. 3

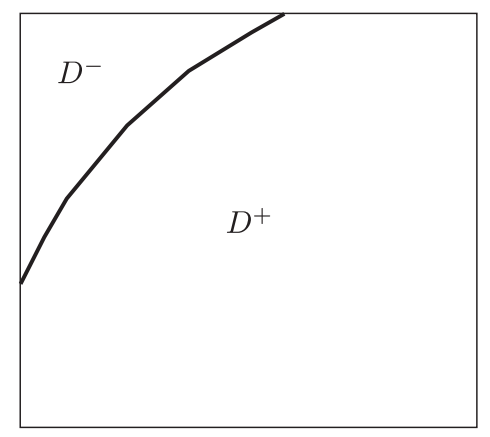

Рис. 5

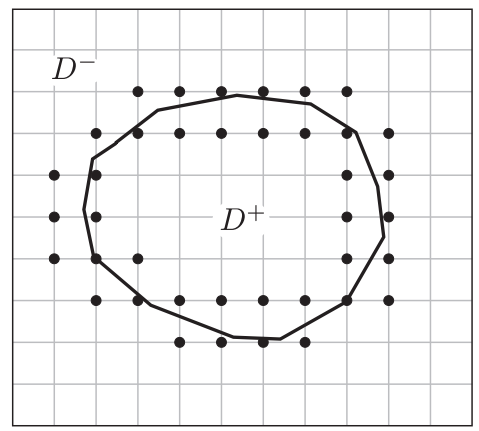

Рис. 2

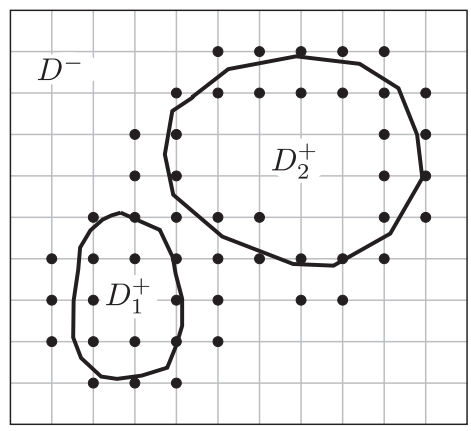

Рис. 4

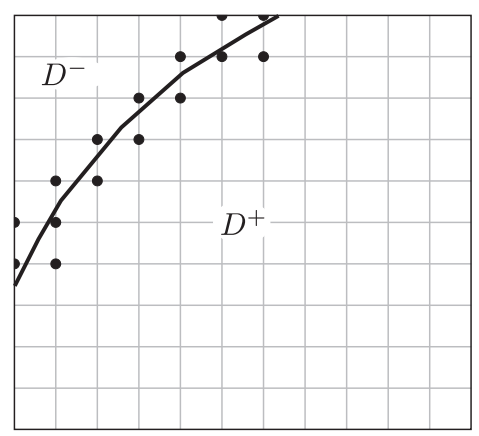

Рис. 6 
На рис. 2, 4 и 6 изображена граница $\gamma$ для каждого из трех сделанных нами выборов подмножеств $M^{+} \subset M$.

Рассмотрим еще четвертый пример, связанный с той же разностной задачей, рассмотренной в примере 1 из раздела 1 . В этом четвертом примере отнесем к $M^{+}$все те и только те точки $m=\left(m_{1} h, m_{2} h\right), m \in M$, каждая из которых лежит внутри квадрата $D$ или на какой-нибудь из сторон этого квадрата, причем $m_{1}+m_{2}-$ четное число.

Очевидно, что $M^{+}$и $M^{-}$будут расположены в квадрате $D$ подобно черным и белым полям шахматной доски.

В этом случае $N^{+}=N^{-}=M$, причем все эти три множества совпадают с границей $\gamma=N^{+} \cap N^{-}$между $N^{+}$и $N^{-}$. Построенный формализм охватывает и этот случай разностной задачи, но содержательность теряется.

\section{3. Определение и свойства разностных потенциалов}

Здесь мы построим разностные потенциалы, которые играют для абстрактных разностных схем (1.1) роль, аналогичную той роли, которую классические интегралы типа Коши играют для аналитических функций или, что то же самое, для решений системы Коши-Римана.

Определим матричную функцию $G_{n m}$ как такую функцию двух аргументов $m \in M$ и $n \in N$, для которой при любой правой части $f_{M} \in F_{M}$ уравнения (1.1) решение $u_{N}=\left\{u_{n}\right\}, n \in N$, может быть записано в виде

$$
u_{n}=\sum_{m \in M} G_{n m} f_{m}, \quad n \in N .
$$

Существование и единственность функции Грина $G_{n m}$ следуют, очевидно, из требования к (1.1) иметь одно и только одно решение $u_{N} \in V_{N}$ при любой $f_{M} \in F_{M}$. Обозначим через $V_{\gamma}$ линейное пространство всех функций $\gamma$, каждая из которых является сужением какой-либо $u_{N} \in V_{N}$, на сеточную границу $\gamma \subset N$. Пусть $v_{\gamma}$ есть какой-нибудь элемент пространства $V_{\gamma}$, которое будем называть пространством плотностей.

ОПРЕДЕЛЕНИЕ 1. Разностными потенциалами $v_{N}^{+}=P_{N \gamma}^{+} v_{\gamma}$ и $v_{N}^{-}=P_{N \gamma}^{-} v_{\gamma}$ с плотностью $v_{\gamma}$ назовем соответственно решения следующих двух задач:

$$
\begin{gathered}
\sum_{n \in N_{m}} a_{m n} v_{n}^{+}= \begin{cases}0, & \text { если } m \in M^{+}, \\
\sum_{n \in N_{m}} a_{m n}\left[\theta_{N}(\gamma) v_{n}\right], & \text { если } m \in M^{-},\end{cases} \\
\sum_{n \in N_{m}} a_{m n} v_{n}^{-}= \begin{cases}\sum_{n \in N_{m}} a_{m n}\left[\theta_{N}(\gamma) v_{n}\right], & \text { если } m \in M^{+}, \\
0, & \text { если } m \in M^{-} .\end{cases}
\end{gathered}
$$

В (3.1) и (3.2) использовано обозначение

$$
\theta_{N}(\gamma) v_{n} \equiv \begin{cases}\left.v_{\gamma}\right|_{n}, & \text { если } n \in \gamma, \\ 0, & \text { если } n \in N \backslash \gamma\end{cases}
$$


ЗАмЕчАНиЕ 1. Заметим, что правая часть формулы (3.1) может отличаться от нуля только в тех точках $m \in M^{-}$, для которых пересечение множеств $N_{m}$ и $\gamma$ непусто: $N_{m} \cap \gamma \neq \varnothing$. Аналогично, правая часть формулы (3.2) может отличаться от нуля в тех точках $m \in M^{+}$, для которых пересечение множеств $N_{m}$ и $\gamma$ непусто: $N_{m} \cap \gamma \neq \varnothing$.

Очевидно, что функции $v_{N}^{+}=\left\{v_{n}^{+}, n \in N\right\}$ и $v_{N}^{-}=\left\{v_{n}^{-}, n \in N\right\}$ могут быть записаны в виде

$$
\begin{array}{ll}
v_{n}^{+}=\sum_{m \in M^{-}} G_{n m}\left(\sum_{n \in N_{m}} a_{m n}\left[\theta_{N}(\gamma) v_{n}\right]\right), & n \in N, \\
v_{n}^{-}=\sum_{m \in M^{+}} G_{n m}\left(\sum_{n \in N_{m}} a_{m n}\left[\theta_{N}(\gamma) v_{n} t\right]\right), & n \in N .
\end{array}
$$

Правые части формул (3.3) и (3.4) суть суммы по тем $m \in M^{-}$или $m \in M^{+}$ соответственно, для которых шаблон $N_{m}$ пересекается с сеточной границей $\gamma$.

ЗАмЕчАниЕ 2. Вычисления разностного потенциала $v_{N}^{+}=P_{N \gamma}^{+} v_{\gamma}$ и разностного потенциала $v_{N}^{-}=P_{N \gamma}^{-} v_{\gamma}$ с плотностью $v_{\gamma}$ можно осуществить, численно решая уравнение (3.1) или уравнение (3.2). Использование формул (3.3) или (3.4) для этого не требуется.

Теорема 1. Имеет место равенство

$$
v_{N}^{+}+v_{N}^{-}=\theta_{N}(\gamma) v_{N}
$$

¿de

$$
\left.\theta_{N}(\gamma) v_{\gamma}\right|_{n}= \begin{cases}v_{n}, & \text { если } n \in \gamma \\ 0, & \text { если } n \in N \backslash \gamma\end{cases}
$$

ДокАЗАТЕЛЬство. Введем обозначение $v_{N}=v_{N}^{+}+v_{N}^{-}$и сложим почленно левые и правые части уравнений (3.1) и (3.2). Получим уравнение

$$
\sum_{n \in N_{m}} a_{m n} v_{n}=\sum_{n \in N_{m}} a_{m n}\left[\theta_{N}(\gamma) v_{n}\right], \quad m \in M=M^{+} \cup M^{-} .
$$

Очевидно, что подстановка функции $\theta_{N}(\gamma) v_{n}$ вместо $v_{n}$ в левую часть уравнения (3.6) обратит это уравнение в тождество. Ввиду единственности решения $v_{N}$ задачи (3.6), являющейся частным случаем задачи (1.1), получаем равенство (3.5).

Обозначим $V_{\gamma}^{+}$линейное подпространство пространства плотностей $V_{\gamma}$, отнеся к $V_{\gamma}^{+}$те и только те элементы $v_{\gamma}^{+}$пространства $V_{\gamma}$, которые можно доопределить всюду на $N^{+} \backslash \gamma$ до некоторой функции $v_{N^{+}}^{+}=\left\{v_{n}^{+}, n \in N^{+}\right\}$, удовлетворяющей однородному уравнению

$$
\sum_{n \in N_{m}} a_{m n} v_{n}^{+}=0, \quad m \in M^{+} .
$$


Обозначим $V_{\gamma}^{-}$линейное подпространство пространства плотностей $V_{\gamma}$, отнеся к $V_{\gamma}^{-}$те и только те функции $v_{\gamma} \in V_{\gamma}$, которые можно доопределить всюду на $N^{-}$до некоторой функции $v_{N^{-}}^{-}$, удовлетворяющей однородному уравнению

$$
\sum_{n \in N_{m}} a_{m n} v_{n}^{-}=0, \quad m \in M^{-} .
$$

ОПРЕДЕЛЕниЕ 2. Введем операторы $P_{\gamma}^{+}: V_{\gamma} \rightarrow V_{\gamma}$ и $P_{\gamma}^{-}: V_{\gamma} \rightarrow V_{\gamma}$, определив их равенствами

$$
\begin{aligned}
& v_{\gamma}^{+}=P_{\gamma}^{+} v_{\gamma}=\left.P_{N \gamma}^{+} v_{\gamma}\right|_{\gamma}, \\
& v_{\gamma}^{-}=P_{\gamma}^{-} v_{\gamma}=\left.P_{N \gamma}^{-} v_{\gamma}\right|_{\gamma} .
\end{aligned}
$$

Теорема 2. Вектор-функиия (или, короче, функция) $v_{\gamma} \in V_{\gamma}$, принадлежит $V_{\gamma}^{+}$в том и только том случае, если она удовлетворяет условию

$$
P_{\gamma}^{+} v_{\gamma}=v_{\gamma}
$$

Если условие (3.11) выполнено, то восполнение $v_{N^{+}}$, удовлетворяющее условию (3.7), единственно и совпадает на $N^{+} \subset N$, с разностным потенииалом $v_{N}^{+}=P_{N \gamma}^{+} v_{\gamma}$ :

$$
v_{n}^{+}=\left.P_{N \gamma}^{+} v_{\gamma}\right|_{n}, \quad \text { если } n \in N^{+} .
$$

Если $v_{\gamma}$ удовлетворяет условию (3.11), то разностный потенииал $v_{N}^{+}=$ $P_{N \gamma}^{+} v_{\gamma}$ с плотностью $v_{\gamma}$ обращается в нуль на сеточной подобласти $N \backslash N^{+}=$ $N^{-} \backslash \gamma$ области $N$ :

$$
\left.P_{N \gamma}^{+} v_{\gamma}\right|_{n}= \begin{cases}v_{n}^{+}, & \text {если } n \in N^{+}, \\ 0, & \text { если } n \in N \backslash N^{+}=N^{-} \backslash \gamma\end{cases}
$$

ДокАЗАтЕЛЬСтво. Пусть $v_{\gamma} \in V_{\gamma}^{+}$, т. е. пусть существует восполнение функции $v_{\gamma}, v_{N^{+}}^{+}=\left\{v_{n}^{+}, n \in N^{+}\right\}$, удовлетворяющее равенству (3.7). Доопределим функцию $v_{N^{+}}^{+}$всюду на $N \backslash N^{+}$до функции $v_{N}^{+}$нулем, положив

$$
v_{n}^{+}= \begin{cases}\left.v_{N^{+}}^{+}\right|_{n}, & \text { если } n \in N^{+}, \\ 0, & \text { если } n \in N \backslash N^{+} .\end{cases}
$$

Покажем, что функция $v_{N}^{+}$, заданная равенством (3.14), совпадает с разностным потенциалом $P_{N \gamma}^{+} v$, т. е. что (3.14) можно записать в виде

$$
P_{N \gamma}^{+} v_{\gamma}^{+}= \begin{cases}\left.v_{N^{+}}^{+}\right|_{n}, & \text { если } n \in N^{+}, \\ 0, & \text { если } n \in N \backslash N^{+} .\end{cases}
$$

Тогда, рассматривая (3.15) лишь в точках $n \in \gamma$, увидим, что условие (3.11) выполнено. Кроме того, тогда будет доказано также, что если восполнение $v_{N^{+}}^{+}$, удовлетворяющее условию (3.7), существует, то оно единственно, а именно совпадает с $P_{N \gamma}^{+} v_{\gamma}$ на $N^{+} \subset N$. 
Итак, докажем, что левая часть формулы (3.14) совпадает с $P_{N \gamma}^{+} v_{\gamma}$. В соответствии с определением $P_{N \gamma}^{+} v_{\gamma}$ для этого надо проверить, что функция (3.14) при заданном $v_{\gamma} \in V_{\gamma}^{+}$удовлетворяет уравнению (3.1). Убеждаемся в этом: подставляя (3.14) в левую часть (3.1) и принимая во внимание (3.7), получаем тождество.

Покажем, наконец, что в случае, если $v_{\gamma}$ удовлетворяет условию $(3.11), v_{\gamma}$ принадлежит $V_{\gamma}^{+}$, т. е. что в этом случае существует восполнение $v_{\gamma}$ всюду на $N^{+}$до функции $v_{N^{+}}^{+}$, удовлетворяющей однородному уравнению (3.7). Таким восполнением является сужение $v_{N^{+}}^{+}$потенциала $v_{N}^{+}=P_{N \gamma}^{+} v_{\gamma}$ на $N^{+} \subset N$. Действительно, это сужение $v_{N^{+}}^{+}$удовлетворяет условию (3.7) в силу вида правой части уравнения (3.1), решением которого является $v_{N}^{+}$. Далее, сужение $v_{\gamma}^{+}=P_{\gamma}^{+} v_{\gamma}$ на $\gamma \subset N^{+}$, в силу (3.11) совпадает на $\gamma$ с $v_{\gamma}$ и, тем самым, $v_{N}^{+}$ является восполнением $v_{\gamma}$ всюду на $N$ и, в частности, на $N^{+} \subset N$. Теорема доказана.

ТЕОРема 3. Вектор-функиия (или, короче, функиия) $v_{\gamma}$ принадлежит $V_{\gamma}^{-}$ в том и только том случае, если она удовлетворяет условию

$$
P_{\gamma}^{-} v_{\gamma}=v_{\gamma}
$$

Если условие (3.16) выполнено, то восполнение $v_{N^{-}}$, удовлетворяющее условию (3.8), единственно и совпадает на $N^{-} \subset N$ с разностным потенииалом $v_{N}^{-}=P_{N \gamma}^{-} v_{\gamma}$ :

$$
v_{N}^{-}=\left.P_{N \gamma}^{-} v_{\gamma}\right|_{n}, \quad \text { если } n \in N^{-} .
$$

Если $v_{\gamma}$ удовлетворяет условию (3.16), то разностный потенииал $v_{N}^{-}=$ $P_{N \gamma}^{-} v_{\gamma}$ с плотностъю $v_{\gamma}$ обращается в нуль на сеточной подобласти $N \backslash N^{-}=$ $N^{+} \backslash \gamma$ сеточной области $N$ :

$$
\left.P_{N \gamma}^{-} v_{\gamma}\right|_{n}= \begin{cases}v_{n}, & \text { если } n \in N^{-}, \\ 0, & \text { если } n \in N \backslash N^{-} .\end{cases}
$$

ДокАЗАтельство. Доказательство совпадет с доказательством теоремы 2, если в последнем всюду поменять в верхнем индексе плюс на минус и, кроме того, вместо уравнения (3.1) использовать уравнение (3.2).

Tеорема 4. Onераторы $P_{\gamma}^{+}$u $P_{\gamma}^{-}$суть проекторы, m.e.

$$
\left(P_{\gamma}^{+}\right)^{2}=P_{\gamma}^{+}, \quad\left(P_{\gamma}^{-}\right)^{2}=P_{\gamma}^{-} ;
$$

выполнено равенство

$$
P_{\gamma}^{+}+P_{\gamma}^{-}=E_{\gamma},
$$

где $E_{\gamma}$ - единичный оператор: $E_{\gamma} v_{\gamma} \equiv v_{\gamma} ;$ имеют место следующие равенства между линейными пространствами:

$$
\begin{array}{ccc}
\operatorname{Im} P_{\gamma}^{+}=V_{\gamma}^{+} & u & \operatorname{Im} P_{\gamma}^{-}=V_{\gamma}^{-}, \\
\operatorname{Ker} P_{\gamma}^{+}=V_{\gamma}^{-} & u & \operatorname{Ker} P_{\gamma}^{-}=V_{\gamma}^{+} .
\end{array}
$$


ДокАзАтЕльство. Докажем первое из равенств (3.18). В соответствии со стандартным определением проектора надо показать, что для любой $v_{\gamma} \in V_{\gamma}$ имеет место равенство

$$
\left(P_{\gamma}^{+}\right)^{2} v_{\gamma}=P_{\gamma}^{+} v_{\gamma}
$$

Обозначим $v_{\gamma}^{+}$функцию $P_{\gamma}^{+} v_{\gamma}$. Тогда (3.22) примет вид

$$
P_{\gamma}^{+} v_{\gamma}^{+}=v_{\gamma}^{+} \text {. }
$$

Но $v_{\gamma}^{+}$является сужением на $\gamma$ разностного потенциала $P_{N \gamma}^{+} v_{\gamma}=v_{N}^{+}$, так что $v_{N}^{+}$является восполнением $v_{\gamma}^{+}$всюду на $N$ и, в частности, на $N^{+} \subset N$. Это восполнение удовлетворяет условию (3.7) в силу структуры правой части уравнения (3.1). Поэтому в силу определения подпространства $V_{\gamma}^{+}$имеет место включение $v_{\gamma}^{+} \in V_{\gamma}^{+}$. Но в силу теоремы 2 элемент $v_{\gamma}^{+}$удовлетворяет условию (3.11), которое совпадает с доказываемым равенством (3.23). Второе равенство (3.18) доказывается аналогично.

Докажем второе утверждение теоремы, т. е. равенство (3.19). Оно означает, что для любой $v_{\gamma}$ имеет место равенство

$$
P_{\gamma}^{+} v_{\gamma}+P_{\gamma}^{-} v_{\gamma}=v_{\gamma}, \quad v_{\gamma} \in V_{\gamma},
$$

которое можно записать в виде

$$
v_{\gamma}^{+}+v_{\gamma}^{-}=v_{\gamma}
$$

Но равенство (3.25) имеет место в силу теоремы 1 и получается сужением левой и правой частей тождества (3.5) с сеточной области $N$ на ее сеточную подобласть $\gamma, \gamma \subset N$.

Докажем теперь первое из двух равенств (3.20). В силу стандартного определения $\operatorname{Im} P_{\gamma}^{+}$надо показать, что для каждой $v_{\gamma} \in V_{\gamma}$ имеет место включение $P_{\gamma}^{+} v_{\gamma} \in V_{\gamma}^{+}$, а также что для каждого элемента $v_{\gamma}^{+} \in V_{\gamma}^{+}$найдется такой элемент $v_{\gamma} \in V_{\gamma}$, для которого выполнено равенство $P_{\gamma} v_{\gamma}=v_{\gamma}^{+}$. В силу доказанного выше для любой $v_{\gamma} \in V_{\gamma}$ имеет место включение $P_{\gamma}^{+} v_{\gamma}=v_{\gamma}^{+} \in V_{\gamma}^{+}$, и, кроме того, любая $v_{\gamma}^{+} \in V_{\gamma}^{+}$, получается из некоторой $v_{\gamma} \in V_{\gamma}$ при преобразовании $P_{\gamma}^{+}: V_{\gamma} \rightarrow V_{\gamma}$; такой функцией $v_{\gamma}$ может служить сама функция $v_{\gamma}^{+} \in V_{\gamma}^{+} \subset V_{\gamma}$, поскольку в силу теоремы 2 для любой $v_{\gamma}^{+} \in V_{\gamma}^{+}$выполнено равенство $P_{\gamma}^{+} v_{\gamma}^{+}=v_{\gamma}^{+}$. Первое равенство (3.20) доказано; второе доказывается аналогично.

Докажем первое из утверждений (3.21) теоремы. Равенство Ker $P_{\gamma}^{+}=V_{\gamma}^{-}$ в развернутом виде означает, что для всех $v_{\gamma}^{-} \in V_{\gamma}^{-}$и только для них имеет место равенство $P_{\gamma}^{+} v_{\gamma}^{-}=0_{\gamma}$. Но в силу уже доказанных второго равенства $(3.20)$ и равенства $(3.25)$

$$
P_{\gamma}^{+} v_{\gamma}^{-}=v_{\gamma}^{-}-P_{\gamma}^{-} v_{\gamma}^{-}=v_{\gamma}^{-}-v_{\gamma}^{-}=0_{\gamma} .
$$

Второе равенство (3.21) доказывается аналогично. Теорема доказана.

ОПРЕДЕЛЕНИЕ 3. Проекторы $P_{\gamma}^{+}: V_{\gamma} \leftarrow V_{\gamma}$ и $P_{\gamma}^{-}: V_{\gamma} \leftarrow V_{\gamma}$ будем называть граничными проекторами. 
Теорема 5. Каждый элемент $v_{\gamma} \in V_{\gamma}$ может быть представлен в виде сумми

$$
v_{\gamma}=v_{\gamma}^{+}+v_{\gamma}^{-}, \quad v_{\gamma}^{+} \in V_{\gamma}^{+}, \quad v_{\gamma}^{-} \in V_{\gamma}^{-},
$$

причем представление (3.27) единственно, т.е. $V_{\gamma}=V_{\gamma}^{+} \oplus V_{\gamma}^{-}$, или $V_{\gamma}$ есть прямая сумма подпространств $V_{\gamma}^{+} u V_{\gamma}^{-}$. Кроме того, имеют место следующие формуль для $u_{\gamma}^{+} u u_{\gamma}^{-}$:

$$
v_{\gamma}^{+}=P_{\gamma}^{+} v_{\gamma}, \quad v_{\gamma}^{-}=P_{\gamma}^{-} v_{\gamma} .
$$

ДокАЗАтЕльство. В силу равенства (3.24) элемент $v_{\gamma} \in V_{\gamma}$ можно представить в виде

$$
v_{\gamma}=P_{\gamma}^{+} v_{\gamma}+P_{\gamma}^{-} v_{\gamma}=v_{\gamma}^{+}+v_{\gamma}^{-}
$$

где

$$
v_{\gamma}^{+}=P_{\gamma}^{+} v_{\gamma}, \quad v_{\gamma}^{-}=P_{\gamma}^{-} v_{\gamma} .
$$

Далее, поскольку $\operatorname{Im} P_{\gamma}^{+}=V_{\gamma}^{+}, \operatorname{Im} P_{\gamma}^{-}=V_{\gamma}^{-}$, имеют место включения $v_{\gamma}^{+} \in$ $V_{\gamma}^{+}, v_{\gamma}^{-} \in V_{\gamma}^{-}$. Таким образом, существование представления (3.27) доказано.

Остается доказать единственность представления (3.27). Ввиду линейности достаточно показать, что в случае $v_{\gamma}=0_{\gamma}$ не существует нетривиального представления $v_{\gamma}$ в виде (3.27).

Допустим противное, и пусть

$$
0_{\gamma}=v_{\gamma}^{+}+v_{\gamma}^{-}
$$

$v_{\gamma}^{+} \in V_{\gamma}^{+}, v_{\gamma}^{-} \in V_{\gamma}^{-}$, и хотя бы одно из слагаемых $v_{\gamma}^{+}$или $v_{\gamma}^{-}$отлично от $0_{\gamma}$. Поскольку $v_{\gamma}^{-} \in V_{\gamma}^{-}$, то в силу (3.29) следует, что $v_{\gamma}^{+}=-v_{\gamma}^{-} \in V_{\gamma}^{-}$. Таким образом, $v_{\gamma}^{+}$принадлежит как $V_{\gamma}^{+}$, так и $V_{\gamma}^{-}$. Точно так же $v_{\gamma}^{-} \in V_{\gamma}^{-}$и $v_{\gamma}^{-} \in V_{\gamma}^{+}$. Покажем теперь, что если какая-либо $z_{\gamma} \in V_{\gamma}$ принадлежит одновременно и $V_{\gamma}^{+}$ и $V_{\gamma}^{-}$, то $z_{\gamma}=0$. В самом деле, функцию $z_{\gamma}$ можно доопределить на $N^{+}$до $z_{N^{+}}$ так, что выполнено

$$
\sum_{n \in N_{M}} a_{m n} z_{n}=0, \quad \text { если } m \in M^{+} \text {. }
$$

Эту же функцию $z_{\gamma}$ можно доопределить на $N^{-}$до некоторой $z_{N}$ так, что будет выполняться

$$
\sum_{n \in N_{m}} a_{m n} z_{n}=0, \quad \text { если } m \in M^{-} \text {. }
$$

Таким образом, существует доопределение $z_{\gamma}$ всюду на $N$, при котором $z_{N}$ удовлетворяет как уравнению (3.30), так и уравнению (3.31), т. е. $z_{N}$ является решением уравнения (3.1) с нулевой правой частью. Поэтому ввиду единственности решения задачи (3.1) справедливо равенство $z_{N}=0_{N}$. Поскольку $\gamma \subset N$, то также $z_{\gamma}=0_{\gamma}$. Теорема доказана. 
Теорема 6. Рассмотрим семейство разностных схем вида (3.1) с различньми множествами $M, N_{m}, N$ и различными коэфбициентами $a_{m n}, m \in M$, $n \in N_{m}$, однако с одними и теми же для всех схем семейства подмножествами $M^{+}$u $N_{m}, m \in M^{+}$, коэфбициентами $a_{m n}, m \in M^{+}, n \in N_{m}$, границей $\gamma u$ пространством плотностей $V_{\gamma}$. Тогда для всех схем семейства подпространство $V_{\gamma}^{+} \subset V_{\gamma}$ будет одним и тем же. Одним и тем же останется также критерий (3.11) того, что справедливо включение $v_{\gamma} \in V_{\gamma}^{+}$.

ДокАзАТЕльство. В силу определения уравнения (3.7), это уравнение будет одним и тем же для всех разностных схем семейства. Поэтому линейное подпространство $V_{\gamma}^{+}$тех элементов $u_{\gamma}^{+}$, которые можно доопределить всюду на $N^{+}$до решения уравнения (3.7), не изменится. Следовательно, в силу теоремы 4 не изменится $\operatorname{Im} P_{\gamma}^{+}$. Тем самым, не изменится и пространство решений уравнения (3.11), так как в силу теоремы 2 выполнение уравнения (3.11) есть критерий справедливости включения $v_{\gamma} \in V_{\gamma}^{+}$. Теорема доказана.

Следует подчеркнуть, однако, что сам проектор $P_{\gamma}^{+}: V_{\gamma} \rightarrow V_{\gamma}$ будет различным для различных схем из описанного выше семейства разностных схем вида (3.1).

ТеОРема 7. Формулировка теоремы 6 останется справедливой, если всюду заменить в верхнем индексе знак "плюс" на знак "минус".

ДокАЗАТЕльство. Следует воспользоваться доказательством теоремы 6 , заменив в этом доказательстве плюс на минус.

ТеОрема 8. Пусть $v_{N}$ - произволъный элемент пространства $V_{N}$. Рассмотрим разностную схему вида (3.1)

$$
\sum_{n \in N_{m}} a_{m n} w_{n}=f_{m}, \quad m \in M,
$$

со следующей правой частью $f_{M}=\left\{f_{m}\right\}, m \in M$ :

$$
f_{m}= \begin{cases}0, & \text { если } m \in M^{+} \\ \sum_{n \in N_{m}} a_{m n} v_{n}, & \text { если } m \in M^{-}\end{cases}
$$

Решение $w_{N}$ задачи (3.32) с правой частъю (3.33) всюду на $N^{+}$не завиcuт от значений $v_{n}$ в точках $n \notin \gamma$ и всюду на $N^{+}$совпадает с разностным потенииалом $v_{N}^{+}=P_{N \gamma}^{+} v_{\gamma}$, т.е. с решением задачи

$$
\sum_{n \in N_{m}} a_{m n} v_{n}^{+}= \begin{cases}0, & \text { если } m \in M^{+} \\ \sum_{n \in N_{m}} a_{m n}\left[\theta_{N}(\gamma) v_{n}\right], & \text { если } m \in M^{-} .\end{cases}
$$

ДокАЗАтЕльство. В силу определения множества $N^{-}$имеют место включения $N_{m} \in N^{-}$для всех $m \in M^{-}$. Поэтому

$$
f_{m}=\sum_{n \in N_{m}} a_{m n}\left[\theta_{N}\left(N^{-}\right) v_{n}\right], \quad \text { если } m \in M^{-} .
$$


Далее, очевидно, что в точках $n \in N^{-}$имеет место равенство

$$
\theta_{N}\left(N^{-}\right) v_{n}=\theta_{N}(\gamma) v_{n}+\theta_{N}\left(N \backslash N^{+}\right) v_{n}
$$

Поэтому в точках $m \in M^{-}$справедливо следующее представление второй строчки формулы (3.33):

$$
\begin{aligned}
& \sum_{n \in N_{m}} a_{m n} v_{n}=\sum_{n \in N_{m}} a_{m n}\left\{\left[\theta_{N}(\gamma) v_{n}\right]+\theta_{N}\left[\left(N \backslash N^{+}\right) v_{n}\right]\right\} \\
& =\sum_{n \in N_{m}} a_{m n}\left[\theta_{N}(\gamma) v_{n}\right]+\sum_{n \in N_{m}} a_{m n}\left[\theta_{N}\left(N \backslash N^{+}\right) v_{n}\right], \quad \text { если } m \in M^{-} .
\end{aligned}
$$

В силу формулы (3.36) и линейности задачи (3.32) функция $w_{N}$ есть сумма решений задачи

$$
\sum_{n \in N_{m}} a_{m n} v_{n}^{+}= \begin{cases}0, & \text { если } m \in M^{+}, \\ \sum_{n \in N_{m}} a_{m n}\left[\theta_{N}(\gamma) v_{n}\right], & \text { если } m \in M^{-},\end{cases}
$$

и задачи

$$
\sum_{n \in N_{m}} a_{m n} z_{n}= \begin{cases}0, & \text { если } m \in M^{+}, \\ \sum_{n \in N_{m}} a_{m n}\left[\theta_{N}\left(N \backslash N^{+}\right) v_{n}\right], & \text { если } m \in M^{-} .\end{cases}
$$

В силу того, что для $m \in M^{+}$справедливо включение $N_{m} \subset N^{+}$, правую часть (3.38) можно записать в виде

$$
\sum_{n \in N_{m}} a_{m n}\left[\theta_{N}\left(N \backslash N^{+}\right) v_{n}\right], \quad m \in M=M^{+} \cup M^{-} .
$$

Теперь видно, что функция $z_{N}=\left\{z_{n}, n \in N\right\}$, задаваемая формулой

$$
z_{n}=\theta_{N}\left(N \backslash N^{+}\right) v_{n}, \quad n \in N=N^{+} \cup N^{-},
$$

при подстановке в уравнение (3.38) обращает это уравнение в тождество, т. е. функция

$$
z_{n}= \begin{cases}0, & \text { если } n \in N^{+}, \\ v_{n}, & \text { если } n \in N \backslash N^{+}=N^{-} \backslash \gamma,\end{cases}
$$

является решением задачи (3.38).

Решение $v_{N}^{+}$задачи (3.37) по определению есть разностный потенциал

$$
v_{N}^{+}=P_{N \gamma}^{+} v_{\gamma} .
$$

Складывая формулы (3.40) и (3.41) почленно, получаем

$$
w_{n}=v_{n}^{+}+z_{n}= \begin{cases}v_{n}^{+}, & \text {если } n \in N^{+}, \\ v_{n}^{+}+v_{n}, & \text { если } n \in N \backslash N^{+}=N^{-} \backslash \gamma,\end{cases}
$$

что доказывает утверждение теоремы. 
ТЕОРема 9. Пусть $v_{\gamma}^{+}$- произвольный элемент подпространства $V_{\gamma}^{+}$, $v_{\gamma}^{+} \in V_{\gamma}^{+} u v_{N}^{+}=P_{N \gamma}^{+} v_{\gamma}^{+}$. Тогда

$$
\left.P_{N \gamma}^{-} v_{\gamma}^{+}\right|_{n}= \begin{cases}-v_{n}^{+}, & \text {если } n \in N^{+} \backslash \gamma \\ 0, & \text { если } n \in N^{-}\end{cases}
$$

Пусть $v_{\gamma}^{-}-$произвольный элемент подпространства $V_{\gamma}^{-}, v_{\gamma}^{-} \in V_{\gamma}^{-} u v_{N}^{-}=$ $P_{N \gamma}^{-} v_{\gamma}^{-}$. Тогда

$$
\left.P_{N \gamma}^{+} v_{\gamma}^{-}\right|_{n}= \begin{cases}0, & \text { если } n \in N^{+} \\ -v_{n}^{-}, & \text {если } n \in N \backslash N^{+}=N^{-} \backslash \gamma\end{cases}
$$

ДокАзАтельство. Докажем утверждение (3.43). В силу равенства (3.5) из теоремы 1 , с учетом теоремы 2 в случае $v_{\gamma} \in V_{\gamma}^{+}$имеют место соотношения $v_{\gamma}=v_{\gamma}^{+}$

$$
P_{N \gamma}^{+} v_{\gamma}^{+}+P_{N \gamma}^{-} v_{\gamma}^{+}=\theta_{N}(\gamma) v_{\gamma}^{+}
$$

Отсюда с учетом (3.13) получим равенство

$$
\left.P_{N \gamma}^{-} v_{\gamma}^{+}\right|_{n}=\left.\theta_{N}(\gamma) v_{\gamma}^{+}\right|_{n}-\left.P_{N \gamma}^{+} v_{\gamma}^{+}\right|_{n}= \begin{cases}-v_{n}^{+}, & \text {если } n \in N^{+} \backslash \gamma, \\ 0_{n}, & \text { если } n \in N^{-},\end{cases}
$$

которое совпадает с равенством (3.43). Второе утверждение теоремы, т. е. равенство (3.44), доказывается аналогично. Теорема доказана.

Наряду с задачей (3.1) рассмотрим задачу

$$
\sum_{n \in N_{m}} a_{m n} u_{n}^{+}=\theta_{m}\left(M^{-}\right) f_{m}, \quad \text { если } \quad m \in M=M^{+} \cup M^{-},
$$

и задачу

$$
\sum_{n \in N_{m}} a_{m n} u_{n}^{-}=\theta_{m}\left(M^{+}\right) f_{m}, \quad \text { если } \quad m \in M=M^{+} \cup M^{-} .
$$

Решение задачи (3.1) есть, очевидно, сумма решений $u_{N}^{+}$и $u_{N}^{-}$задач $(3.45)$ и $(3.46)$ :

$$
u_{N}=u_{N}^{+}+u_{N}^{-} .
$$

Слагаемое $u_{N}^{+}$есть вклад в $u_{N}$ влияния источников $f_{m}$, локализованных в точках $m \in M^{-}$, а $u_{N}^{-}-$вклад источников $f_{m}$, локализованных в точках $m \in M^{+}$.

Теорема 10. Вклад $u_{N}^{+}$источников $f_{m}, m \in M^{-}$, локализованных на $M^{-}$, в решение $u_{n}$ задачи (3.1) в точках $n \in N^{+}$совпадает с разностным потенциалом $v_{N}^{+}=P_{N \gamma}^{+} u_{\gamma}$ с плотностью $u_{\gamma}$, т.е. имеет место равенство

$$
u_{n}^{+}=v_{n}^{+}=\left.P_{N \gamma}^{+} u_{\gamma}\right|_{n}, \quad \text { если } n \in N^{+} .
$$

Вклад $u_{N}^{-}$источников $f_{m}, m \in M^{+}$, локализованных на $M^{+}$, в решение $u_{n}$ задачи (3.1) в точках $n \in N^{-}$совпадает с разностным потенииалом $v_{N}^{-}=$ $P_{N \gamma}^{-} u_{\gamma}$ с плотностью $u_{\gamma}$, m.е. имеет место равенство

$$
u_{n}^{-}=v_{n}^{-}=\left.P_{N \gamma}^{-} u_{\gamma}\right|_{n}, \quad \text { если } n \in N^{-} .
$$


ДоказАтельство. Справедливость теоремы есть непосредственное следствие теоремы 8.

Наряду с $P_{N \gamma}^{+} v_{\gamma}$ и $P_{N \gamma}^{-} v_{\gamma}$ введем тесно связанные с ними разностные потенциалы $P_{N \gamma}^{ \pm} v_{\gamma}$ и $P_{N \gamma}^{\mp} v_{\gamma}$, положив

$$
\begin{aligned}
& v_{N}^{ \pm}=P_{N \gamma}^{ \pm} v_{\gamma}=\left\{\begin{array}{ll}
\left.P_{N \gamma}^{+} v_{\gamma}\right|_{n}, & n \in N^{+}, \\
-\left.P_{N \gamma}^{-} v_{\gamma}\right|_{n}, & n \in N^{-}
\end{array},\right. \\
& v_{N}^{\mp}=P_{N \gamma}^{\mp} v_{\gamma}=-P_{N \gamma}^{ \pm} v_{\gamma}, \quad n \in N \text {. }
\end{aligned}
$$

Потенциалы (3.49) и (3.50) можно записать подробнее в виде

$$
\begin{aligned}
& v_{n}^{ \pm}= \begin{cases}v_{n}^{+}, & n \in N^{+}, \\
-v_{n}^{-}, & n \in N^{-},\end{cases} \\
& v_{n}^{\mp}= \begin{cases}v_{n}^{-}, & n \in N^{-}, \\
-v_{n}^{+}, & n \in N^{+} .\end{cases}
\end{aligned}
$$

Разностные потенциалы $v_{N}^{ \pm}$и $v_{N}^{\mp}$ суть функции, определенные на $N=$ $N^{+} \cup N^{-}$и двузначные в точках $n \in \gamma=N^{+} \cap N^{-}$.

ОПРЕДЕЛЕНИЕ 4. Функции вида

$$
w_{n}^{ \pm}= \begin{cases}w_{n}^{+}, & n \in N^{+}, \\ -w_{n}^{-}, & n \in N^{-},\end{cases}
$$

где $w_{N^{+}}^{+}=\left\{w_{n}^{+}, n \in N^{+}\right\}$и $w_{N^{-}}^{-}=\left\{w_{n}^{-}, n \in N^{-}\right\}-$какие-нибудь решения соответственно уравнений (3.7) и (3.8):

$$
\sum_{n \in N_{m}} a_{m n} w_{n}^{+}=0, \quad m \in M^{+}, \quad \sum_{n \in N_{m}} a_{m n} w_{n}^{-}=0, \quad m \in M^{+},
$$

будем называть кусочно регулярными решениями однородного уравнения, соответствующего уравнению (1.1). Функцию

$$
v_{\gamma}=\left[w_{N}^{ \pm}\right]=w_{\gamma}^{+}-w_{\gamma}^{-}
$$

будем называть скачком кусочно регулярного решения $w_{N}^{ \pm}$на границе $\gamma$.

ТЕОРема 11. Разностный потенииал $v_{N}^{ \pm}=P_{N \gamma}^{ \pm} v_{\gamma}$ с плотностью $v_{\gamma}$ есть кусочно регулярное решение уравнения (1.1) со скачком $v_{\gamma},\left[v_{N}^{ \pm}\right]_{\gamma}=v_{\gamma}$, на сеточной гранище $\gamma$.

ДокАЗАТЕльство. В силу теоремы 2 сужение $v_{N^{+}}^{+}=\left.P_{N \gamma}^{+} v_{\gamma}\right|_{N^{+}}$потенциала $P_{N \gamma}^{+} v_{\gamma}$ с плотностью $v_{\gamma}$ удовлетворяет уравнению (3.7). Аналогично, сужение $v_{N^{-}}^{-}=\left.P_{N \gamma}^{-} v_{\gamma}\right|_{N^{-}}$в силу той же теоремы 2 удовлетворяет уравнению (3.8). Таким образом, разностный потенциал $P_{N \gamma}^{ \pm} v_{\gamma}$ есть кусочно регулярное решение, скачок

$$
\left[P_{N \gamma}^{ \pm} v_{\gamma}\right]=\left.P_{N \gamma}^{+} v_{\gamma}\right|_{\gamma}+\left.P_{N \gamma}^{-} v_{\gamma}\right|_{\gamma}=P_{\gamma}^{+} v_{\gamma}+P_{\gamma}^{-} v_{\gamma}
$$

которого в силу теоремы 1 совпадает с плотностью $v_{\gamma}$. 
ТЕОРема 12. Пусть $v_{\gamma}-$ произвольный элемент пространства плотностей $V_{\gamma}, v_{\gamma} \in V_{\gamma}$. Существует одно и только одно кусочно регулярное решение уравнения (1.1), имеющее этот скачок.

ДокАзАтЕльство. Существование доказано тем, что функция $v_{N}^{ \pm}=P_{N \gamma}^{ \pm} v_{\gamma}$, заданная равенством (3.49), в силу (3.5) является кусочно регулярным решением со скачком $\gamma$. Остается доказать единственность.

В виду линейности достаточно установить, что в случае $v_{\gamma}=0_{\gamma} \in V_{\gamma}$ единственным кусочно регулярным решением является функция

$$
w_{n}^{ \pm}= \begin{cases}0_{n}, & n \in N^{+}, \\ 0_{n}, & n \in N^{-} .\end{cases}
$$

Установим это. Кусочно-регулярное решение (3.49) с нулевым скачком $\left[w_{N}^{ \pm}\right]_{\gamma}=w_{\gamma}^{+}-w_{\gamma}^{-}=0_{\gamma}$ есть однозначная на границе $\gamma$ функция, так что $w_{N}^{ \pm} \in V_{N}$. В то же время эта функция удовлетворяет как уравнению (3.7), так и уравнению (3.8), т. е. удовлетворяет однородному уравнению вида (1.1)

$$
\sum_{n \in N_{m}} a_{m n} w_{n}^{ \pm}=0, \quad m \in M^{+} \cup M^{-}=M .
$$

Но в силу единственности решения задачи (1.1) из (3.55) следует, что $w_{n}^{ \pm} \equiv 0$.

\section{4. Аналогия между разностными потенциалами и интегралами Коши}

Имеет место глубокая аналогия между разностными потенциалами с плотностью на сеточном контуре $\gamma$ и интегралами Коши с плотностью на несамопересекающемся гладком замкнутом контуре $Г$, разделяющем комплексную плоскость $D$ на ограниченную подобласть $D^{+}$и ее дополнение $D^{-}$.

Для выяснения этой аналогии предварительно изложим некоторые известные сведения об интегралах типа Коши в форме, удобной для сравнения этих интегралов и их свойств с разностными потенциалами и их свойствами.

Обозначим $V_{\Gamma}$ линейное пространство всех комплекснозначных функций $v_{\Gamma}(\xi)$ аргумента $\xi \in \Gamma$, удовлетворяющих некоторым условиям гладкости.

Запишем интегралы Коши $u_{D}^{+}=P_{D \Gamma}^{+} v_{\Gamma}$ и $u_{D}^{-}=P_{D \Gamma}^{-} v_{\Gamma}$ с плотностью $v_{\Gamma} \in V_{\Gamma}$, в следующем виде:

$$
\begin{aligned}
& u_{D}^{+}(z)= \begin{cases}\frac{1}{2 \pi i} \oint \frac{v_{\gamma}(\xi)}{\xi-z} d \xi, & \text { если } z \notin \gamma, \\
\frac{1}{2 \pi i} \lim _{z_{0} \rightarrow z} \oint \frac{v_{\Gamma}(\xi)}{\xi-z_{0}} d \xi, & \text { если } z \in \Gamma, z_{0} \in D^{+},\end{cases} \\
& u_{D}^{-}(z)= \begin{cases}\frac{1}{2 \pi i} \oint \frac{v_{\gamma}(\xi)}{\xi-z} d \xi, & \text { если } z \notin \gamma, \\
\frac{1}{2 \pi i} \lim _{z_{0} \rightarrow z} \oint \frac{v_{\Gamma}(\xi)}{\xi-z_{0}} d \xi, & \text { если } z \in \gamma, z_{0} \in D^{-} .\end{cases}
\end{aligned}
$$


В формуле (4.1) интегрирование ведется с обходом Г против часовой стрелки, а в формуле (4.2) обход Г ведется по часовой стрелке. Известно, что имеет место равенство

$$
\left.\left(u_{D}^{+}+u_{D}^{-}\right)\right|_{z}= \begin{cases}0, & \text { если } z \notin \Gamma, \\ v_{\Gamma}(z), & \text { если } z \in \Gamma .\end{cases}
$$

Запишем граничные проекторы Коши $P_{\Gamma}^{+}: V_{\Gamma} \rightarrow V_{\Gamma},\left(P_{\Gamma}^{+}\right)^{2}=P_{\Gamma}^{+}$, и $P_{\Gamma}^{-}$: $V_{\Gamma} \rightarrow V_{\Gamma},\left(P_{\Gamma}^{-}\right)^{2}=P_{\Gamma}^{-}$, следующим образом:

$$
\begin{aligned}
& \left.P_{\Gamma}^{+} v_{\Gamma}\right|_{\xi \in \Gamma}=\lim u_{D}^{+}(z), \quad z \rightarrow \xi, \quad z \in D^{+}, \\
& \left.P_{\Gamma}^{-} v_{\Gamma}\right|_{\xi \in \Gamma}=\lim u_{D}^{+}(z), \quad z \rightarrow \xi, \quad z \in D^{-},
\end{aligned}
$$

или короче:

$$
\begin{aligned}
& v_{\Gamma}^{+}=P_{\Gamma}^{+} v_{\Gamma}=\left.P_{D \Gamma}^{+} v_{\Gamma}\right|_{\Gamma}, \\
& v_{\Gamma}^{-}=P_{\Gamma}^{-} v_{\Gamma}=\left.P_{D \Gamma}^{-} v_{\Gamma}\right|_{\Gamma} .
\end{aligned}
$$

Обозначим через $V_{\Gamma}^{+}$и $V_{\Gamma}^{-}$соответственно линейные подпространства всех тех $v_{\Gamma}, v_{\Gamma} \in V_{\Gamma}$, которые являются граничными значениями аналитических внутри $D^{+}$функций или граничными значениями аналитических внутри $D^{-}$ функций, стремящихся к нулю при $z \rightarrow \infty$. Справедливо утверждение, что $v_{\Gamma}^{+} \in V_{\Gamma}^{+}$и $v_{\Gamma}^{-} \in V_{\Gamma}^{-}$в том и только том случае, если выполнены следующие сингулярные интегральные соотношения:

$$
P_{\Gamma}^{+} v_{\Gamma}^{+}=v_{\Gamma}^{+} \quad \text { и } \quad P_{\Gamma}^{-} v_{\Gamma}^{-}=v_{\Gamma}^{-}
$$

соответственно. Если условия (4.6) выполнены, то соответствующие аналитические функции $u_{D^{+}}^{+}(z), z \in D^{+}$, и $u_{D^{-}}^{-}(z), z \in D^{-}$, совпадают на $D^{+}$и $D^{-}$ соответственно с интегралами Коши (4.1), (4.2). При этом имеют место формулы:

$$
\begin{aligned}
& \left.P_{D \Gamma}^{+} v_{\Gamma}^{+}\right|_{z}= \begin{cases}v_{D}^{+}(z), & \text { если } z \in D^{+} \cup \Gamma, \\
0, & \text { если } z \in D^{-},\end{cases} \\
& \left.P_{D \Gamma}^{+} v_{\Gamma}^{-}\right|_{z}= \begin{cases}0, & \text { если } z \in D^{+} \cup \Gamma, \\
-v_{D^{+}}^{+}(z), & \text { если } z \in D^{-},\end{cases} \\
& \left.P_{D \Gamma}^{-} v_{\Gamma}^{-}\right|_{z}= \begin{cases}v_{D}^{-}(z), & \text { если } z \in D^{-} \cup \Gamma, \\
0, & \text { если } z \in D^{+},\end{cases} \\
& \left.P_{D \Gamma}^{-} v_{\Gamma}^{+}\right|_{z}= \begin{cases}0, & \text { если } z \in D^{-} \cup \Gamma, \\
-v_{D}^{+}(z), & \text { если } z \in D^{+} .\end{cases}
\end{aligned}
$$

Введем обозначение

$$
u_{D}^{ \pm}(z)= \begin{cases}u_{D^{+}}^{+}(z), & z \in D^{+} \cup \Gamma, \\ -u_{D^{-}}^{-}(z), & z \in D^{-} \cup \Gamma,\end{cases}
$$


где $u_{D^{+}}^{+}(z)$ и $u_{D^{-}}^{-}(z)$ определены формулами (4.1) и (4.2) соответственно. Функция $u_{D}^{ \pm}(z)$ обладает следующим свойством: $u_{D}^{ \pm}(z)$ есть кусочно аналитическая функция, двузначная в точках $z \in \Gamma$, претерпевающая скачок $v_{\Gamma}(\xi)$ при переходе $z$ в точке $\xi \in \Gamma$ через контур $\Gamma$ со стороны области $D^{-}$в область $D^{+}$ на комплексной плоскости и стремящаяся к нулю при $z \rightarrow \infty$. Известно, что наличие этого свойства является характеристическим, так что функцию $u_{D}^{ \pm}(z)$ можно определить не формулой (4.11), а просто как кусочно аналитическую функцию, претерпевающую заданный скачок $v_{\Gamma}(\xi)$ в точках $\xi \in \Gamma$ и стремящуюся к нулю при $z \rightarrow \infty$.

Теперь проследим аналогию между конструкциями, свойствами и ролью для аналитических функций в областях $D^{+}$и $D^{-}$комплексной плоскости интегралов типа Коши с одной стороны и конструкциями, свойствами и ролью разностных потенциалов для решений разностной схемы (1.1) в сеточных областях $N^{+}$ и $N^{-}-$с другой.

$\mathbf{1}^{\circ}$. Прежде всего заметим, что интегралы типа Коши можно интерпретировать как некоторые потенциалы для решений системы дифференциальных уравнений Коши-Римана, связывающей вещественные и мнимые части аналитических функций.

$\mathbf{2}^{\circ}$. Сеточные подобласти $N^{+}$и $N^{-}$сеточной области $N$ аналогичны подобластям $D^{+}$и $D^{-}$комплексной плоскости $D$, а сеточная граница $\gamma=N^{+} \cap N^{-}$ аналогична контуру Г на комплексной плоскости.

$3^{\circ}$. Разностные потенциалы $P_{N \gamma}^{+} v_{\gamma}$ и $P_{N \gamma}^{-} v_{\gamma}$ с плотностью $v_{\gamma} \in V_{\gamma}$, записанные в форме (3.3) и (3.4), аналогичны интегралам типа Коши (4.1) и (4.2).

$4^{\circ}$. Формула (3.5) аналогична формуле (4.3).

$5^{\circ}$. Разностные граничные проекторы $P_{\gamma}^{+}: V_{\gamma} \rightarrow V_{\gamma}$ и $P_{\gamma}^{-}: V_{\gamma} \rightarrow V_{\gamma}$ аналогичны граничным проекторам Коши $P_{\Gamma}^{+}: V_{\Gamma} \rightarrow V_{\Gamma}$ и $P_{\Gamma}^{-}: V_{\Gamma} \rightarrow V_{\Gamma}$, определенным формулами (4.4) и (4.5).

$\mathbf{6}^{\circ}$. Уравнения $(3.11),(3.16)$ относительно $v_{\gamma}^{+}$и $v_{\gamma}^{-}$на сеточной границе $\gamma$ аналогичны сингулярным интегральным уравнениям (4.6) на контуре Г.

$7^{\circ}$. Критерий (3.11) того, что $v_{\gamma}^{+}$является следом на $N^{+}$какого-нибудь кусочно регулярного решения, и критерий (3.16) того, что $v_{\gamma}^{-}$является следом на $N^{-}$какого-нибудь кусочно регулярного решения разностной схемы (1.1), аналогичны соответственно критериям (4.6) того, что $v_{\Gamma}^{+}$и $v_{\Gamma}^{-}$являются граничными значениями какой-нибудь непрерывной на $D^{+} \cup \Gamma$ и аналитической внутри $D^{+}$функции $v^{+}(z)$ или, соответственно, непрерывной на $D^{-} \cup \Gamma$ и аналитической внутри $D^{-}$функции $v^{-}(z)$, стремящейся к нулю при $z \rightarrow \infty$.

$\mathbf{8}^{\circ}$. Формулы (3.13), (3.17), (3.43), (3.44) для разностных потенциалов аналогичны соответственно формулам (4.7), (4.9), (4.10), (4.8) для интегралов типа Коши.

$\mathbf{9}^{\circ}$. Формула $V_{\gamma}=V_{\gamma}^{+} \oplus V_{\gamma}^{-}$, доказанная в теореме 5 , аналогична формуле $V_{\Gamma}=V_{\Gamma}^{+} \oplus V_{\Gamma}^{-}$, имеющей место для аналитических функций.

$\mathbf{1 0}^{\circ}$. Теорема 11 о том, что разностный потенциал $v_{N}^{ \pm}=P_{N \gamma}^{ \pm} v_{\gamma}$ можно равносильно определить как кусочно регулярное решение уравнения (1.1) со скачком $v_{\gamma}$ на сеточном контуре, есть точный аналог того, что интеграл Коши (4.11) можно равносильно определить как кусочно аналитическую функцию с заданным скачком $v_{\Gamma}(\xi)$ на контуре $\Gamma$. 
Список аналогий между разностными потенциалами и интегралами Коши можно продолжить. Но и сказанного достаточно для обоснования утверждения, сделанного в начале этого раздела 4.

ЗАмЕчАниЕ 3. Подчеркнем, что выяснение аналогии между разностными потенциалами и интегралами Коши не используется для обоснования теории разностных потенциалов, однако эта аналогия способствует созданию правильной интуиции при использовании этой теории для приложений.

\section{5. Теория разностных потенциалов как база для некоторых приложений}

Линейные разностные задачи вида (1.1) возникают:

- при разностной аппроксимации линейных стационарных краевых или эволюционных начально-краевых задач;

- при вычислении решений нелинейных стационарных разностных краевых задач методом итераций Ньютона, поскольку на каждом шаге нужно вычислять решение некоторой линейной разностной задачи;

- при решении нелинейных начально-краевых задач по неявным разностным схемам на "верхнем" по времени слое;

- при математическом моделировании задач управления подавлением внешнего шума.

Основу большинства уже реализованных приложений теории разностных потенциалов к вычислительным задачам математической физики и к задачам моделирования подавления внешнего шума в защищаемой подобласти составляют следующие три свойства $1^{*}, 2^{*}, 3^{*}$.

Свойство $1^{*}$ установлено в теореме 2 (раздел 3 ). В силу этого свойства уравнение

$$
\sum_{n \in N_{m}} a_{m n} u_{n}=0, \quad m \in M^{+},
$$

в сеточной подобласти $N^{+} \subset N$, и уравнение

$$
u_{\gamma}-P_{\gamma}^{+} u_{\gamma}=0
$$

на границе $\gamma=N^{+} \cap N^{-}$подобласти равносильны.

Благодаря свойству $1^{*}$ можно осуществлять редукцию задач с дополнительными краевыми условиями для уравнения (5.1) в области к задачам на границе $\gamma$.

Благодаря свойству $1^{*}$ можно также при рассмотрении задач вида

$$
\sum_{n \in N_{m}} a_{m n} u_{n}=f_{m}, \quad m \in M,
$$

равносильно заменять подсистему вида (5.1) системы (5.3) уравнением (5.2). При этом $\gamma$ играет роль искусственной границы, а условие (5.2) - роль искусственных граничных условий. 
Свойство 2* установлено в теореме 6. Пусть задача (1.1) заменена другой задачей того же вида, для которой, однако, остаются неизменными подмножества $M^{+}$и $N_{m}, m \in M^{+}$, коэффициенты $a_{m n}, m \in M^{+}, n \in N_{m}$, граница $\gamma$ и пространство $V_{\gamma}$. Тогда потенциал $P_{N \gamma}^{+} v_{\gamma}, v_{\gamma} \in V_{\gamma}$, и разностный проектор $P_{\gamma}^{+}$, вообще говоря, изменяются, но линейное пространство $V_{\gamma}^{+}$решений задачи (5.2) остается прежним. Для $v_{\gamma} \in V_{\gamma}^{+}$потенциал $P_{N^{+} \gamma} v_{\gamma}$ также остается прежним.

Благодаря свойству $2^{*}$ при редукции уравнения (5.1) к уравнению (5.2) на границе $\gamma$, а также при использовании (5.2) в качестве искусственного граничного условия можно воспользоваться допустимой свободой в выборе схемы (1.1), чтобы получить удобное для работы уравнение (5.2).

Свойство $3^{*}$ установлено в теореме 5 . Оно состоит в том, что по сумме $u_{\gamma}=$ $u_{\gamma}^{+}+u_{\gamma}^{-}$влияний $u_{\gamma}^{+}$и $u_{\gamma}^{-}$соответственно источников $f_{m}, m \in M^{+}$, и $f_{m}$, $m \in M^{-}$, можно судить о каждом вкладе отдельно, а именно

$$
u_{\gamma}^{+}=P_{\gamma}^{+} u_{\gamma}, \quad u_{\gamma}^{-}=P_{\gamma}^{-} u_{\gamma} .
$$

Свойство $3^{*}$ позволяет строить управление подавлением внешнего шума в точках защищаемой подобласти $N^{-}$от нежелательного влияния (шума) внешних источников $f_{m}, m \in M^{+}$.

\section{6. Библиографическая справка}

Теория разностных потенциалов, аналогичных интегралам Коши, была предложена автором в 1969 г. в его докторской диссертации и существенно развита в последующие годы автором, А. Я. Белянковым, Д. С. Каменецким, М. И. Лазаревым, М.Н. Мишковым, А. А. Резником, И. Л. Софроновым, В. А. Торгашовым, В.И. Турчаниновым, С. В. Утюжниковым, С. В. Цынковым - см. работы [1]-[23] и монографии [24], [25], где имеется также библиография оригинальных работ, относящихся к теме и принадлежащих многим авторам.

Назовем основные направления, в которых к настоящему времени получены приложения теории.

Алгоритмы численного решения некоторых краевых задач математической физики путем редукции на сеточную границу расчетной подобласти, не требующие разностной аппроксимации граничных условий, разработаны А. Я. Белянковым, К. В. Брушлинским, А. В. Воронковым, А. В. Забродиным, Е. В. Зиновьевым, Н. М. Зуевой, Д. С. Каменецким, М. Ю. Лохановым, М. С. Михайловой, М. Н. Мишковым, В.В.Огневой, Б. З. Оссеровичем, А. А. Резником, И. Л. Софроновым, Е. П. Сычуговой, В. А. Торгашовым, Н. Б. Тузовой, В. И. Турчаниновым и автором (см. [26]-[34]).

Серия работ по нелокальным искусственным граничным условиям при расчете обтекания тела параллельным потоком набегающего газа выполнена C.В. Цынковым. Обзор этих работ, написанный по просьбе автора самим C. В. Цынковым, составляет главу 2 части IV книги [25], см. также [36]-[39].

Задачу о замене уравнений приграничного градиентного слоя при течении газа около стенки нелокальными искусственными граничными условиями на самой стенке поставил и рассмотрел С. В. Утюжников [40], [41]. 
Работы по точной замене волнового уравнения, а также системы уравнений Максвелла вне ограниченной расчетной подобласти неотражающими искусственными граничными условиями выполнены В. И. Турчаниновым, С. В. Цынковым и автором [42]-[46]. Аналогичная работа для системы Максвелла в собственном времени выполнена С. В. Петропавловским [47].

Способ синтеза алгоритмов для решения краевых задач в составных областях, не требующий согласования сеток в подобластях и разностной аппроксимации условий состыковки на границах между подобластями, предложен в [48].

Ряд работ В. С. Рябенького, Р. И. Вейцмана, Е. В. Зиновьева, С. В. Утюжникова, С. В. Цынкова и других авторов посвящен активному управлению подавлением внешнего шума в защищаемой подобласти. См. [49]-[59] и имеющуюся там библиографию. На основе использования разностных потенциалов построена также теория управления решениями абстрактных линейных разностных схем в составных областях [60], [61].

\section{Список литературы}

[1] В. С. Рябенький, Суммы для решений разностных уравнений, аналогичные интегралу Коши для аналитических функиий, и некоторые приложения этих сумм, Деп. В ВИНИТИ, №635-69, М., 1969.

[2] В. С. Рябенький, Некоторые вопросы теории разностных краевых задач, Дис. ... докт. физ.-матем. наук, М., 1969.

[3] В. С. Рябенький, "Оператор граничного проектирования", Докл. АН СCCP, 185:3 (1969), 521-523; англ. пер.: V.S. Ryaben'kii, "A boundary projection operator", Soviet Math. Dokl, 10 (1969), 385-387.

[4] В. С. Рябенький, "О системах разностных уравнений на полиэдрах", Матем. сб., 79(121):1(5) (1969), 78-90; англ. пер.: V. S. Ryaben'kii, "On systems of difference equations on polyhedra", Math. USSR-Sb., 8:1 (1969), 73-85.

[5] В. С. Рябенький, "Метод внутренних граничных условий в теории разностных краевых задач", УМH, 26:3(159) (1971), 105-160; англ. пер.: V.S. Ryaben'kii, "The method of intrinsic boundary conditions in the theory of difference boundary value problems", Russian Math. Surveys, 26:3 (1971), 117-176.

[6] А.Я. Белянков, K развитию метода внутренних граничных условий в теории разностных схем, Дис. ... канд. физ.-матем. наук, МФТИ, М., 1977.

[7] В. С. Рябенький, А. Я. Белянков, "Разностные потенциалы и проекторы", Докл. AH CCCP, 254:5 (1980), 1080-1084; англ. пер.: V. S. Ryaben'kii, A. Ya. Belyankov, "Difference potentials and projectors", Soviet Math. Dokl., 22:2 (1980), 536-540.

[8] А.Я. Белянков, В.С. Рябенький, Проекторы для разностных схем на нерегулярных сетках, Препринт ИПМ им. М.В. Келдыша АН СССР №70, М., 1981, $11 \mathrm{c}$.

[9] А.А. Резник, “Аппроксимация поверхностных потенциалов эллиптических операторов разностными потенциалами”, Докл. АН СССР, 263:6 (1982), 1318-1320; англ. пер.: А. A. Reznik, "Approximation of surface potentials of elliptic operators by difference potentials", Soviet Math. Dokl., 25:2 (1982), 543-545.

[10] А.А. Резник, Аппроксимация поверхностных потенциалов эллиптических операторов разностными и решение краевых задач, Дис. ... канд. физ.-матем. наук, МФТИ, М., 1983.

[11] И.Л. Софронов, Развитие метода разностных потенциалов и применение его $\kappa$ решению стационарных задач дифракции, Дис. ... канд. физ.-матем. наук., МФТИ, М., 1984. 
[12] И. Л. Софронов, "Новый метод численного решения регулярных эллиптических задач", Докл. АН СССР, 296:5 (1987), 1057-1061; англ. пер.: I. L. Sofronov, "A new method of numerical solution of regular elliptic problems", Soviet Math. Dokl., 36:2 (1988), 361-365.

[13] В. С. Рябенький, “Обобщенные проекторы Кальдерона и граничные уравнения на основе концепции четкого следа", Докл. АН CCCP, 270:2 (1983), 288-292; англ. пер.: V.S. Ryaben'kii, "Generalization of Calderón projectors and boundary equations on the basis of the sharp trace concept", Soviet Math. Dokl., 27:3 (1983), 600-604.

!!

[14] В.С. Рябенький, "Проекторы Кальдерона для системы Стокса", УМН, 38:5 (1983), 157-158.

[15] В. С. Рябенький, "Граничные уравнения с проекторами”, УМН, 40:2(242) (1985), 121-149; англ. пер.: V.S. Ryaben'kii, "Boundary equations with projections", Russian Math. Surveys, 40:2 (1985), 147-183.

[16] В. С. Рябенький, "Потенциалы для общих линейных систем разностных уравнений на абстрактных сетках", Журн. вычисл. матем. и матем. физ., 36:4 (1996), 41-49; англ. пер.: V. S. Ryaben'kii, "Potentials for general linear systems of difference equations on abstract grids", Comput. Math. Math. Phys., 36:4 (1996), 451-456.

[17] В.С. Рябенький, "Кусочно-регулярные решения разностных уравнений и разностные потенциалы с плотностью из пространства скачков", Докл. PАН, 347:2 (1996), 160-163; англ. пер.: V.S. Ryaben'kii, "Piecewise regular solutions of difference equations, and difference potentials with density from a space of jumps", Dokl. Math., 53:2 (1996), 183-186.

[18] Д.С. Каменецкий, "Разностные потенциалы и параметризация решений однородных разностных уравнений", Журн. вычисл. матем. и матем. физ., 38:11 (1998), 1829-1843; англ. пер.: D.S. Kamenetskii, "Difference potentials and the parametrization of solutions of homogeneous difference equations", Comput. Math. Math. Phys., 38:11 (1998), 1754-1767.

[19] Д. С. Каменецкий, "Разностные обобщенные операторы Пуанкаре-Стеклова и потенциалы с плотностью из пространства скачков", Журн. вычисл. матем. и матем. физ., 39:8 (1999), 1328-1336; англ. пер.: D. S. Kamenetskii, "Difference generalized Poincaré-Steklov operators and potentials with density from a space of jumps", Comput. Math. Math. Phys., 39:8 (1999), 1275-1282.

[20] V.S. Ryaben'kii, "Difference potentials method and its application", Math. Nachr., 177:1 (1996), 251-264.

[21] А. А. Резник, В. С. Рябенький, И. Л. Софронов, В. И. Турчанинов, "Об алгоритме метода разностных потенциалов", ЖҚрн. вычисл. матем. и матем. физ., 25:10 (1985), 1496-1505; англ. пер.: A. A. Reznik, V.S. Ryaben'kii, I. L. Sofronov, V.I. Turchaninov, "The algorithm of the method of difference potentials", U.S.S.R. Comput. Math. Math. Phys., 25:5 (1985), 144-151.

[22] Е. В. Зиновьев, Решение внешних задач для уравнения Гельмгольца. Приложение $\kappa$ расчету осесимметричных элементов машин, Дис. ... канд. физ.-матем. наук, ИПМ им. М. В. Келдыша АН СССР, М., 1990.

[23] Д. С. Каменецкий, В. С. Рябенький, С. В. Цынков, Граничные уравнения с проекторами в составных областях, Препринты ИПМ им. М. В. Келдыша АН СССР № 112, 113, М., 1991.

[24] В. С. Рябенький, Метод разностных потенииалов и его приложения, 2-е изд., Физматлит, М., 2002, 494 с.; англ. пер. 2-го изд.: V.S. Ryaben'kii, Method of difference potentials and its applications, Springer Ser. Comput. Math., 30, Springer-Verlag, Berlin, 2002, xviii+538 pp. 
[25] В. С. Рябенький, Метод разностных потенииалов и его приложения, Физматлит, М., 2010.

[26] А. В. Забродин, В.В. Огнева, Решение уравнения теплопроводности в областях сложной структуры, Препринт ИПМ им. М. В. Келдыша АН СССР №21, М., 1973, 22 c.

[27] А.Я. Белянков, А.А. Резник, В.С. Рябенький, Численное решение уравнений метода разностных проекторов, Препринт ИПМ им. М. В. Келдыша АН СССР № 105, М., 1981, 27 с.

[28] В. С. Рябенький, И. Л. Софронов, "Численное решение пространственных внешних задач для уравнений Гельмгольца методом разностных потенциалов", Численное моделирование в аэрогидродинамике, Наука, М., 1986, 187-201.

[29] М. Ю. Лоханов, "Численное решение задачи Трикоми методом разностных потенциалов", Труды ХХХ научно-технической конферениии, МФТИ, М., 1984.

[30] Н. М. Зуева, М. С. Михайлова, В. С. Рябенький, Перенос граничных условий из бесконечности на искусственную границу для разностного уравнения Лапласа, Препринт ИПМ им. М.В.Келдыша АН СССР №110, М., 1991, 19 с.

[31] К. В. Брушлинский, В. С. Рябенький, Н. Б. Тузова, "Перенос граничного условия через вакуум в осесимметричных задачах", Журн. вычисл. матем. и матем. физ., 32:12 (1992), 1929-1939; англ. пер.: K. V. Brushlinskii, V. S. Ryaben'kii, N. B. Tuzova, "The transfer of boundary conditions across a vacuum in axisymmetric problems", Comput. Math. Math. Phys., 32:12 (1992), 1757-1767.

[32] В. С. Рябенький, В.А. Торгашов, "Метод разностных потенциалов для численного решения внутренней задачи о плоском течении вязкой несжимаемой жидкости", Докл. РАН, 337:4 (1994), 450-453; англ. пер.: V.S. Ryaben'kii, V.A. Torgashov, "The method of difference potentials for the numerical solution of an interior problem of the plane flow of a viscous incompressible fluid", Russian Acad. Sci. Dokl. Math., 50:1 (1995), 108-113.

[33] В. С. Рябенький, В.А. Торгашов, "Безытерационный способ решения неявной разностной схемы для уравнений Навье-Стокса в переменных: завихренность и функция тока", Матем. моделирование, 8:10 (1996), 100-112.

[34] В. А. Торгашов, "Метод разностных потенциалов для численного решения уравнений Навье-Стокса", Матем. моделирование, 10:8 (1998), 81-102.

[35] V.S. Ryaben'kii, S. V. Tsynkov, "Artificial boundary conditions for the numerical solution of external viscous flow poblems", SIAM J. Numer. Anal., 32:5 (1995), $1355-1389$.

[36] S. V. Tsynkov, "Numerical solution of problems on unbounded domains. A review", Appl. Math., 27:4 (1998), 465-532.

[37] S. V. Tsynkov, "External boundary conditions for three-dimensional problems of computational aerodynamics", SIAM J. Sci. Comput., 21:1 (1999), 166-206.

[38] S. V. Tsynkov, S. Abarbanel, J. Nordstram, V. S. Ryaben'kii, V. N. Vatsa, "Global artificial boundary conditions for computation of external flow problems with propulsive jets", Proceedings of the 14th AIAA CFD Conference, v.2, A Collection of Technical Papers (Norfolk, VA, June-July 1999), 1999, 836-846.

[39] V.S. Ryaben'kii, "Nonreflecting time-dependent boundary conditions on artificial boundaries of varying location and shape", Appl. Numer. Math., 33:1-4 (2000), 481-492.

[40] S. V. Utyuzhnikov, "The method of boundary condition transfer in application to modeling near-wall turbulent flows", Comput. ES Fluids, 35:10 (2006), 1193-1204.

[41] S. V. Utyuzhnikov, "Domain decomposition for near-wall turbulent flows", Comput. Es Fluids, 38:9 (2009), 1710-1717. 
[42] В. С. Рябенький, В. И. Турчанинов, С. В. Цынков, "Использование лакун 3D-волнового уравнения для вычисления решения на больших временах", Матем. моделирование, 11:12 (1999), 113-126.

[43] V.S. Ryaben'kii, S. V. Tsynkov, V. I. Turchaninov, "Long-time numerical computation of wave-type solutions driven by moving sources", Appl. Numer. Math., 38:1-2 (2001), 187-222.

[44] В. С. Рябенький, В. И. Турчанинов, С. В. Цынков, "Неотражающие искусственные граничные условия для замены отбрасываемых уравнений с лакунами", $M a-$ тем. моделирование, 12:12 (2000), 108-127.

[45] В.С. Рябенький, В.И. Турчанинов, “Использование лакун гиперболических уравнений и метода разностных потенциалов для расчета дифракции волн в ограниченной окрестности рассеивателя, но на больших временах", Журн. вычисл. матем. и матем. физ., 45:8 (2005), 1435-1449; англ. пер.: V.S. Ryabenkii, V.I. Turchaninov, "The use of lacunas of hyperbolic equations and the difference potential method for computing wave diffraction in a bounded neighborhood of a scatterer at long times", Comput. Math. Math. Phys., 45:8 (2005), 1385-1399.

[46] В. С. Рябенький, В.Н.Турчанинов, "Неотражающие граничные условия на основе использования лакун для вычисления решений системы Максвелла на больших временах", Докл. РАН, 404:1 (2005), 21-24; англ. пер.: V.S. Ryabenkii, V.I. Turchaninov, "Lacuna-based nonreflecting artificial boundary conditions for computing solutions to Maxwell's equations over long times", Dokl. Math., 72:2 (2005), 672-675.

[47] С. В. Петропавловский, "Экономный алгоритм расчета длительных электромагнитных явлений в собственном времени в ограниченной расчетной области", Журн. вычисл. матем. и матем. физ., 45:10 (2005), 1826-1836; англ. пер.: S. V. Petropavlovsky, "Efficient algorithm for proper-time computations of long electromagnetic phenomena in a bounded computational domain", Comput. Math. Math. Phys., 45:10 (2005), 1759-1768.

[48] В. С. Рябенький, В.И. Турчанинов, Е. Ю. Эпштейн, "Схема композиции алгоритмов для задач в составных областях на базе метода разностных потенциалов", ЖКрн. вычисл. матем. и матем. физ., 46:10 (2006), 1853-1870; англ. пер.: V.S. Ryaben'kii, V. I. Turchaninov, Ye. Yu. Epshteyn, "Algorithm composition scheme for problems in composite domains based on the difference potential method", Comput. Math. Math. Phys., 46:10 (2006), 1768-1784.

[49] В.С. Рябенький, "Разностная задача экранирования", Функи. анализ и его прил., 29:1 (1995), 90-91; англ. пер.: V.S. Ryaben'kii, "Finite-difference shielding problem", Funct. Anal. Appl., 29:1 (1995), 70-71.

[50] Р. Н. Вейцман, В. С. Рябенький, "Разностные задачи экранирования и имитации", Докл. РАН, 354:2 (1997), 151-154; англ. пер.: V.S. Ryaben'kii, R. I. Vejtsman, "Difference problems of screening and simulation", Dokl. Math., 55:3 (1997), 340-343.

[51] V.S. Ryaben'kii, S. V. Tsynkov, S. V. Utyuzhnikov, "Inverse source problem and active shielding for composite domains", Appl. Math. Lett., 20:5 (2007), 511-515.

[52] H. Lim, S. V. Utyuzhnikov, Y.W. Lam, A. Turan, M. Avis, V.S. Ryaben'kii, S. V. Tsynkov, "Experimental validation of the active noise control methodology based on difference potentials", AIAA J., 47:4 (2009), 874-884.

[53] V.S. Ryaben'kii, S. V. Tsynkov, S. V. Utyuzhnikov, "Active control of sound with variable degree of cancellation", Appl. Math. Lett., 22:12 (2009), 1846-1851.

[54] S. V. Utyuzhnikov, "Active wave control and generalized surface potentials", Adv. in Appl. Math., 43:2 (2009), 101-112.

[55] В. С. Рябенький, "Идея использования слабого шума для управления подавлением сильного шума в экранируемой подобласти в реальном времени", Докл. РАН, 430:2 (2010), 166-168; англ. пер.: V.S. Ryaben'kii, "The idea of using weak noise 
for the real-time control of strong noise suppression in a shielded subdomain", Dokl. Math., 81:1 (2010), 137-138.

[56] В. С. Рябенький, "Модель активного экранирования заданной подобласти от шума внешних источников в текущем времени", Журн. вычисл. матем. и матем. физ., 51:3 (2011), 480-491; англ. пер.: V.S. Ryaben'kii, "Model of real-time active noise shielding of a given subdomain subject to external noise sources", Comput. Math. Math. Phys., 51:3 (2011), 444-454.

[57] В. С. Рябенький, "Синхронная разведка для управления подавлением внешнего шума в трехмерной подобласти в реальном времени”, Журн. вычисл. матем. и матем. физ., 51:10 (2011), 1889-1904; англ. пер.: V.S. Ryaben'kii, "Synchronous exploration for the control of real-time external noise suppression in a three-dimensional subdomain", Comput. Math. Math. Phys., 51:10 (2011), 1777-1791.

[58] В. С. Рябенький, "Подавление в реальном времени шума в защищаемой подобласти трехмерного пространства на основе информации от синхронной разведки шумом", Докл. РАН, 439:3 (2011), 319-322; англ. пер.: V. S. Ryaben'kii, "Real-time noise suppression of a protected subdomain in three-dimensional space based on information from the synchronous exploration of noise", Dokl. Math., 84:1 (2011), 562-564.

[59] В. С. Рябенький, "Ключевая информация для управления решениями линейных разностных схем в составных областях", Докл. РАН, 444:4 (2012), 376-377.

[60] В. С. Рябенький, Теория управления решениями линейных разностных схем в составных областях, Препринт № 11, ИПМ им. М. В. Келдыша РАН, М., 2012.

[61] В. С. Рябенький, "Математическая модель устройств подавления внешнего шума в подобласти пространства", Матем. моделирование, 24:8 (2012).

B. C. Рябенький (V.S. Ryaben'kii)

Поступила в редакцию

Институт прикладной математики

05.12 .2011

им. М. В. Келдыша РАН

E-mail: ryab@keldysh.ru 